\title{
Appropriate Criterion of Spontaneous Ignition of an Externally Heated Solid Fuel in Numerical Study*
}

\author{
Yuji NAKAMURA** and Tadao TAKENO**
}

\begin{abstract}
A numerical study was made to identify the most appropriate criterion to describe the spontaneous ignition of an externally heated solid material. Several ignition criteria were examined to determine whether they can represent correctly the effects of the gravity and ambient oxygen concentration on the ignition characteristics. Gravity significantly affects the ignition behavior since the buoyancy induced flow plays a key role in the transient mass and heat transfer processes, leading to two distinct types of ignition ${ }^{(1)-(3)}$. Seven ignition criteria, which were used in a 1-D analysis of ignition process, were examined in this study. It was found that some of the criteria based on temperature profiles lead to erroneous predictions of ignition. This is because complicated transport phenomena caused by the buoyancy induced flow control the transient temperature profile, producing a situation where the ignition criteria were apparently satisfied. The present study shows that the criterion based on the local reaction rate is the universal and most appropriate one.
\end{abstract}

Key Words: Spontaneous Ignition, Criterion, Numerical Analysis, Natural Convection, Chemical Reaction, Gas-Phase Ignition

\section{Introduction}

Ignition of solid material induced by irradiated thermal flux is generally considered to be the initial stage of combustion in fires; a well-known but complicated phenomenon. Although the study of this transient process is a quite challenging research topic, the understanding of the process greatly contributes to the prevention of fire disaster on one hand, and to our scientific knowledge on the other. For both reasons, a number of analytical, experimental and numerical studies have been conducted to elucidate the mechanism of the ignition and its behavior. However, there has been little concern about the most appropriate criterion to characterize ignition, especially in numerical studies. Although some empirical ignition criteria have been commonly used in most of numeri-

* Received 23rd October, 2000. Japanese original : Trans. Jpn. Soc. Mech. Eng., Vol.66, No.641, B (2000), pp. 241-248 (Received 8th March, 1999)

** Graduate School of Engineering, Department of Mechanical Engineering, Nagoya University, 1 Furocho, Chikusa-ku, Nagoya 464-8603, Japan. E-mail: yuji@mech.nagoya-u.ac.jp cal studies so far, there seems to be no universal criterion at present.

In general, the instant of ignition in an experiment is defined as the first light emission from the combustible region and detected by a photomultiplier ${ }^{(e x .4)}$ or equivalent device; this judgement is quite reasonable and consistent with our natural sense and experience. This experimental and phenomenological instant must be identified correctly in analytical and numerical studies. In analytical study of thermal explosion theory the ignition is defined by the instant when no steady state solution can exist (Semenov's theory ${ }^{(5),(6)}$ ), and this definition is very clear mathematically. In numerical studies, on the other hand, the instant is usually defined as the time when a certain physical variable (e.g. temperature or reaction rate) exceeds an artificially introduced critical value ${ }^{(7)-(16)}$. Note that in this definition the specified critical values are somewhat arbitrary so that a number of selections could be made. Because of this ambiguity, it is quite possible that one criterion may predict ignition for certain problems but may not for others. In addition, the criterion may be satisfied several times during the 
flaming process. This could be one of the reasons why the predicted ignition characteristics would be inconsistent from one scenario to another. It is our goal to elucidate the numerically-introduced universal ignition criterion which is valid on a general ignition problem.

There are some earlier numerical studies that investigated the most appropriate ignition criterion for a combustible solid material in one-dimensional domain induced by irradiated thermal flux ${ }^{(7)-(9)}$. In these papers, the several criteria which have been widely proposed by several research groups were tested, and it was shown that qualitative behavior of the predicted ignition characteristics are almost independent of the selection of the criterion. Due to this conclusion, there seemed to be no need of further argument about which criterion is the most appropriate one.

However, the recent findings by Nakamura et $\mathrm{al}^{(2),(10)}$ in their 2-D radiation-induced ignition investigation at various gravity levels, reveal that the above conclusion is no longer valid in two-dimensional ignition processes; the qualitative behavior of ignition characteristics for the various gravity levels was found to be different depending on the ignition criterion. This is believed to be caused by the additional multi-dimensional heat and mass transport processes due to the buoyancy-driven flow which was not considered in the one-dimensional studies. Thus, there is still a need to understand what would be an universal and appropriate numerical criterion of ignition for more general ignition problems. To this end, it is essential to understand correctly how the gravity should affect the ignition and why the criterion should affect the qualitative ignition behavior in multidimensional ignition processes.

The present study numerically predicts the detailed processes of irradiation-induced ignition and its behavior at various gravity levels (in a 2-D axisymmetric configuration) and explores which ignition criterion can describe the correct qualitative ignition behavior for a variety of gravity levels between zero $^{-} g$ to normal- $g$. The validity of the criterion is checked by studying the characteristic ignition behavior at various ambient oxygen concentrations from zero to one hundred percent oxygen, because it is well-known that ambient oxygen crucially affects the ignition processes. Seven ignition criteria are arbitrary selected from earlier studies which have been widely used in most analyses of ignition. A detailed discussion is given for the criterion-dependence in the results. In the end, we propose the most appropriate and universal ignition criterion for general ignition problems.

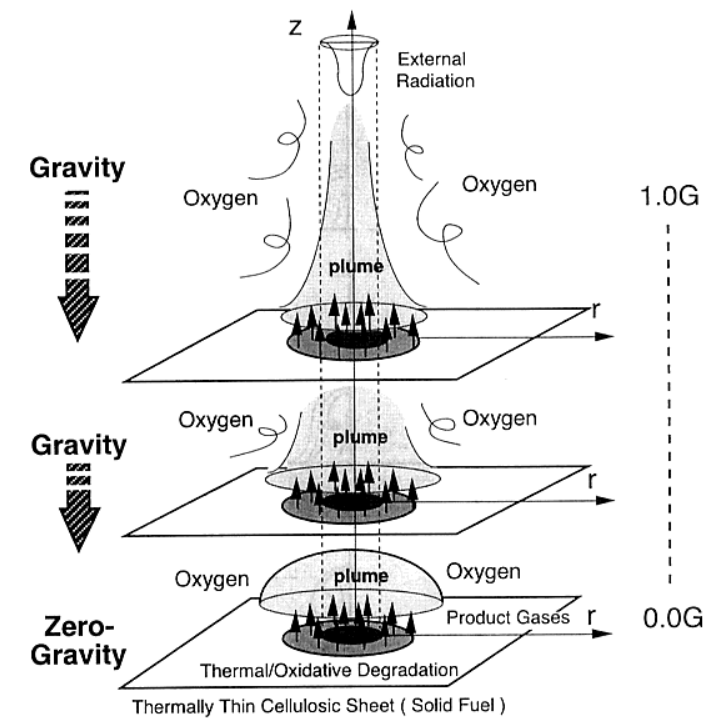

Fig. 1 Schematic model of 2-D ignition

\section{Model Description}

The ignition scenario investigated in this study is in a 2-D axisymmetric physical domain as shown in Fig. 1. The detailed descriptions of the numerical model can be found elsewhere ${ }^{(1),(2)}$ so that a brief description will be given here. A thermally thin combustible solid material is placed horizontally in a quiescent atmosphere. It is then exposed vertically a thermal flux with a Gaussian distribution in the radial direction, which enhances the degradation reaction in the solid material. Decomposed gases including both combustible and non-combustible components are evolved to form a hot-gas plume over the surface. As soon as the gases are evolved in the ambient, the mixing process between the combustible gas components (i.e. fuel gas) and the ambient oxygen starts to form a combustible mixture in the plume over the surface. The heated mixture then ignites spontaneously in the gas-phase. A cylindrical coordinate is employed; the horizontal $(=r)$ and the axial axis $(=$ $z$ ) are placed on the surface and on the center line of the thermal flux, respectively. The equations to be solved in the gas-phase are mass, momentum, energy and species conservation equations, while in the solid-phase the mass, energy and species conservative equations are used ${ }^{(2)}$. The reaction kinetics in the gas-phase is modeled by a one-step over-all reaction, and the solid-phase chemistry is described by three pyrolysis and oxidative reactions ${ }^{(17)}$. The parameters varied in this study are the gravity level and the ambient oxygen concentration. 


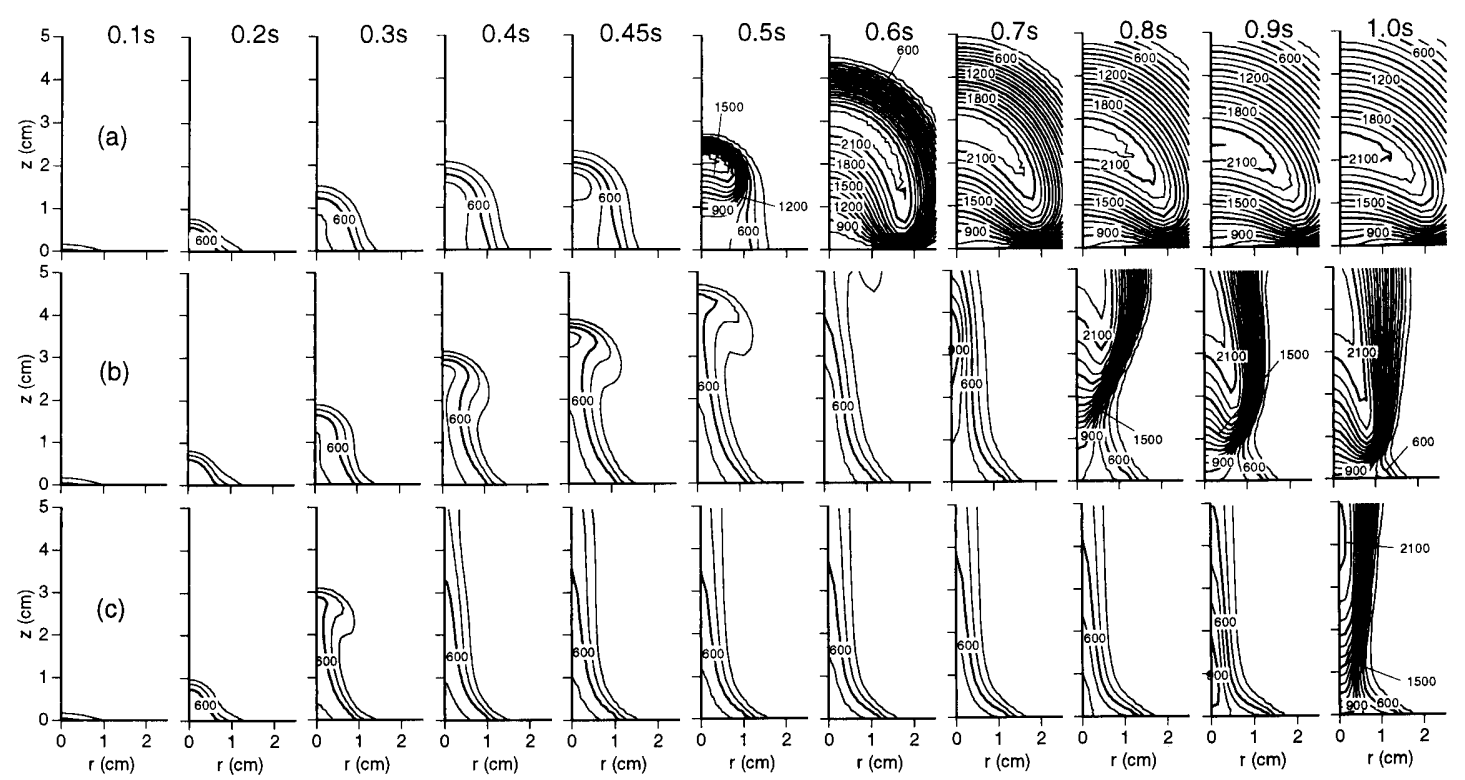

Fig. 2-1 Time-sequences of 2-D temperature distributions under the conditions of ( a ) $g=0.0 \mathrm{G}$, ( b ) $g=0.25 \mathrm{G}$, ( c ) $g=1.0 \mathrm{G}$; and $Y_{a x, \infty}=0.4$

\section{Ignition Characteristics}

\section{1 General requirement}

In general, the ignition is considered to occur locally when heat generation exceeds heat loss to the ambient. The increase in local temperature thus leads to a thermal explosion. In this sense, we have to impose the following two essential requirements for the onset of ignition;

(1) Ignition is a local phenomenon in space and time.

(2) The ignition must lead to stable flame propagation and combustion.

Ignition must be recognized at a local point and at a certain time as the instant of first appearance of thermal wave that develop into stable combustion $^{(\mathrm{ex} .11)}$. Thus, a localized heat generation that does not develop into stable combustion immediately should not be taken as the instant of ignition.

\section{2 Ignition in gravity fields}

In this section we study the gravity dependence of the ignition process through careful observations of the numerical results. Figure 2 shows time-sequences of 2-D temperature and reaction rate distributions for a case when ambient oxygen concentration, $Y_{o x, \infty}$, is 0.4 at three different gravity levels ; (a ) $g=0.0 \mathrm{G}$, ( b ) $g=0.25 \mathrm{G}$, (c ) $g=1.0 \mathrm{G}$, where $\mathrm{G}$ represents the normal gravity $\left(=980.665 \mathrm{~cm} / \mathrm{s}^{2}\right)$. The temperature is shown by contour lines (every $100 \mathrm{~K}$ ) whereas the reaction rate is shown by a shadow plot. Since the distributions of decomposed gases are essentially similar to those of temperature ${ }^{(1),(2)}$, the combustible mixture (composed of the fuel gas in the decomposed gases and oxygen in ambient) is considered to be distributed in the periphery of temperature distributions (around $400-600 \mathrm{~K}$ ).

In Fig. 2-1, comparison of time-sequences of temperature contours at three gravity levels leads to the following conclusions. First, the ignition delay time tends to increase as the gravity level increases. Second, the ignition starts at some position on the symmetry axis irrespective of the gravity. The second happens because the maximum intensity of the incident thermal flux is located on the symmetry axis in the present analysis. The degradation of the solid material to start at a position on the axis, and the subsequent mixing and acceleration of reaction in the gas-phase also starts from that position. Moreover since the radial gradients of temperature and concentrations on the axis of symmetry must vanish, the heat and mass transport there is limited to the axial direction only. Thus, an accumulation of heat at some position on the axis is pronounced; leading to the thermal explosion which is strongly sensitive to the local temperature.

In Fig. 2-2, comparison of detailed timesequences of temperature and reaction rate contours at two different gravity levels ( ( a ) $g=0.0 \mathrm{G}$, ( b ) $g=0.25 \mathrm{G}$ ) just after the ignition event shows the effects of gravity. For the case of zero $-g$ (Fig. 2-2( a )), the combustible mixture is formed in an almost spherical geometry, with the periphery of the hot-gas plume controlled only by molecular diffusion. After the ignition, the thermal wave travels along the mixture from its starting position on the axis toward the solid surface. For the case of $g=0.25 \mathrm{G}$ (Fig. 2-2 




Fig. 2-2 Time-sequences of (1) temperature and (2) reaction rate distributions during the ignition process under the conditions of ( a ) $g=0.0 \mathrm{G}[0.45 \sim$ $0.55 \mathrm{~s}]$, (b) $g=0.25 \mathrm{G}[0.66 \sim 0.76 \mathrm{~s}]$; and $Y_{a x, \infty}=0.4$. Maximum reaction rate is $10^{-3} \mathrm{~g} /\left(\mathrm{cm}^{3} \mathrm{~s}\right)$.

(b )), on the other hand, the hot-gas plume is elongated vertically and squeezed in radial direction by the buoyancy-induced flow; thus enhancing the ambient oxygen penetration into the plume by means of the molecular diffusion. Consequently, the combustible mixture is formed in wider region along the axis than the case of zero $^{-} g^{(1),(2)}$. Although the mixture region and its formation process depend on the gravity level, the behavior of the thermal wave subsequent to the ignition is found to be independent of the gravity. Once the ignition occurs at any position on the axis, the thermal wave travels along the combustible mixture region toward the surface. Note that the time of the first appearance of the thermal wave on the axis (i.e. ignition delay time) and its position change dramatically depending on the gravity level. It is found that the ignition is faster at the plume tip for the case of zero- $g$ whereas the slower ignition inside the plume for the case of $g=0.25 \mathrm{G}$.

Note that both previously-stated-requirements (requirements (1) and (2) above), are always satisfied irrespective of the gravity level. With regard to the specific features of the ignition behavior in various gravity levels, it is seen that there are two distinct types of ignition, 'tip ignition' (ignition occurs at the plume tip) and 'inside ignition' (ignition occurs at inside the plume), depending on the gravity level ${ }^{(1)-(3)}$. The location and the instant of the ignition also depends on the gravity level. These variations should be taken into account to deduce the appropriate criteria for ignition.

In Fig. 2-3, time-sequences of temperature and reaction rate contours at $g=0.25 \mathrm{G}$ during an early ignition event are shown. In this case, a nonmonotonic temperature and reaction rate rise in the vicinity of the plume tip is observed. These quantities first increase with time, but then decrease and eventually die out; the thermal wave is not established immediately. It is suggested that the local heat release near the plume tip is not sufficient to break the local heat balance leading to a thermal runaway during the duration shown in Fig. 2-3 (Note that a thermal wave is finally established inside the plume at a later stage, not at the plume tip, as is seen in Fig. $2-2(\mathrm{~b})$ at around $t=0.7 \mathrm{~s}$ ). The time-dependent energy balance near the plume tip is necessary to determine whether the ignition may occur at the tip or inside the plume; that is the most characteristic feature of the ignition behavior in the presence of gravity. Therefore, allow for the appearance of a nonmonotonic event ('false' ignition) must be excluded by setting an appropriate criterion of ignition. 


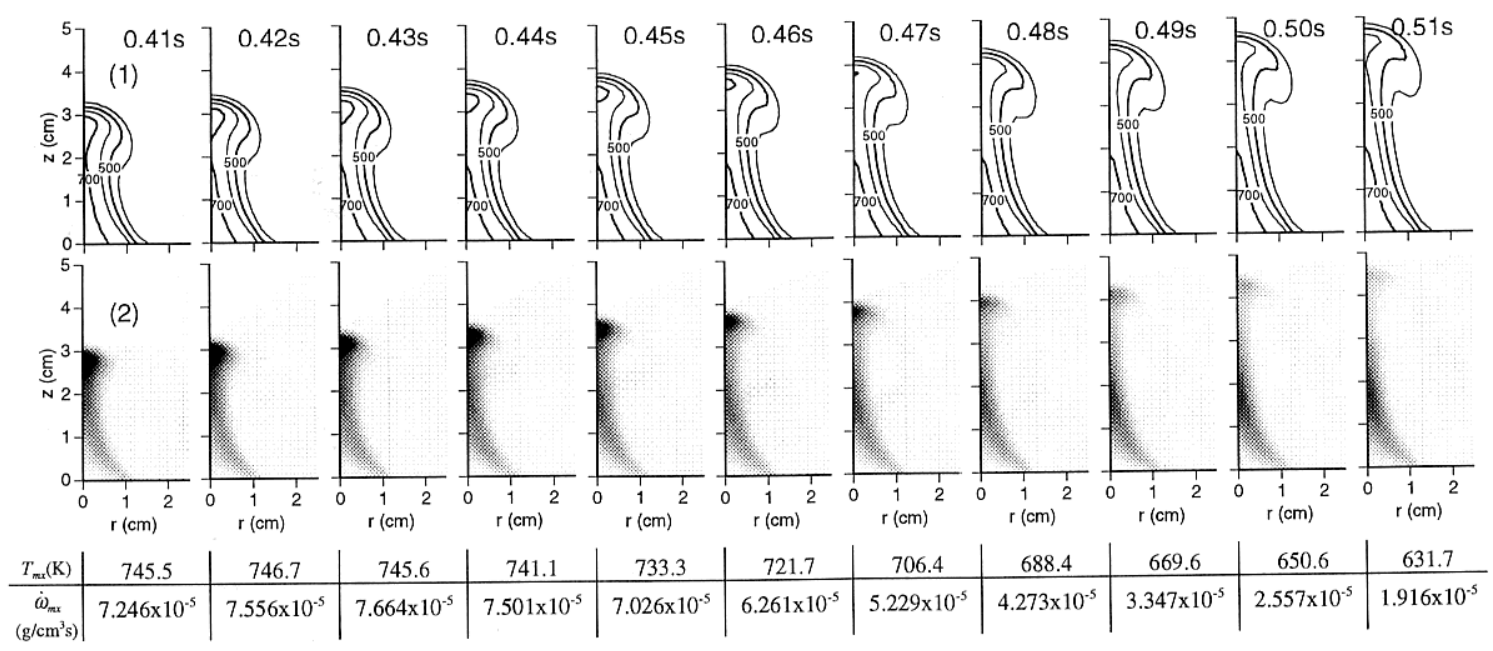

Fig. 2-3 Time-sequences of ( 1 ) temperature and ( 2 ) reaction rate distributions in early ignition event under the conditions of $g=0.25 \mathrm{G}[0.41 \sim 0.51 \mathrm{~s}]$ and $Y_{a x, \infty}=0.4$. Maximum reaction rate is $5 \times 10^{-5} \mathrm{~g} /\left(\mathrm{cm}^{3} \mathrm{~s}\right)$. Table below figures indicates the maximum temperature $\left(T_{m x}\right)$ and reaction rate $\left(\dot{\omega}_{m x}\right)$ at the plume tip.

Finally, it should be noted that two distinct types of ignition have also been found previously in various ambient oxygen concentrations under the presence of gravity $^{(1),(2)}$. It must be emphasized that the appropriate criterion for ignition should distinguish one type from another for a wide range of gravity levels and ambient oxygen concentrations.

To determine whether an actual ignition event in various gravity levels and ambient oxygen concentrations takes place, the following requirements should be added to the two listed above ;

(3) Ignition starts at somewhere on the symmetry axis.

(4) There are two distinct types of ignition ('tip ignition' and 'inside ignition') ; the ignition criterion should correctly distinguish those.

(5) 'False' ignition does exist under certain conditions and the ignition criterion must exclude this possibility.

(6) Ignition delay time decreases as the gravity level increases, whereas the ignition position varies non-monotonically.

(7) Ignition criterion must be independent of gravity and ambient oxygen concentration.

Those seven requirements summarized here are the essential factors for 'true' (not 'false') ignition. If a proposed ignition criterion leads to an any ignition behavior that contradicts those requirements, that criterion must be incorrect.

\section{Test of Ignition Criteria}

The following seven ignition criteria, which have been often used in a 1-D analysis, are tested in this study (references and the physical meaning of each criterion are also described).

Crt. $1: \quad \frac{\partial T_{g}}{\partial z}=0$

(from Ref.(10))

* The instantaneous appearance of thermal peak on the symmetry axis; a local gas element at this position becomes the heat source to the surrounding ambient.

Crt. 2: $T_{g} \geq T_{s} \quad$ (from Ref.(12))

* The temperature of local gas element exceeds the surface temperature; the local gas element becomes a heat source to the surface.

Crt. 3: $\left.\quad \frac{\partial T_{g}}{\partial z}\right|_{\text {on surface }}=0 \quad$ (from Ref. ( 9 ))

* The axial gradient of temperature on the surface becomes zero; the local gas element starts to heat the surface.

Crt. 4: $\quad \dot{\omega}_{g} \geq C_{i g, 4} \quad$ (from Refs. ( 7) and (8))

* The local reaction rate exceeds a certain critical value; sufficient heat starts to be released in the gas element at that position.

Crt. 5: $\quad \int_{V} \dot{\omega}_{g} d V \geq C_{i g, 5}$

(from Refs. ( 7 ), (13) and (14))

* The total heat release rate in the whole region exceeds a certain critical value; sufficient heat starts to be released in the whole region.

Crt. 6: $\quad \frac{\partial T_{g}}{\partial t} \geq C_{i g, 6}$

(from Refs. ( 8 ), (15) and (16))

* The rate of temperature increase at a point exceeds a certain critical value; at that position the temperature start to increase very rapidly. 
Crt. 7: $\quad \frac{\partial \dot{\omega}_{g}}{\partial t} \geq C_{i g, 7}$

(from Ref. ( 7 ))

* The rate of reaction rate increase at a point exceeds a certain critical value; at that position the reaction rate start to increase very rapidly.

Here, $T_{g}, T_{s}$, and $\dot{\omega}_{g}$, respectively represent the gas temperature, the solid surface temperature and the local reaction rate of gas element. The quantity $C_{i g}$ represents a certain numerical value. Since the ignition in this study should occur somewhere on the symmetry axis, see requirement ( 3 ) above, the seven proposed criteria can be evaluated on the axis. This still excludes the fifth proposed criterion, which needs global information. In the following discussion, we simply use the expression 'Crt. $n(n: 1-7)$ ' to represent any of the criterion described above.

In this study, following two important characteristics of the ignition are introduced to discuss the ignition behavior; ignition delay time $\left(t_{i g}\right)$ and ignition position $\left(z_{i g}\right)$. Here, the ignition delay time is defined as the time elapsed from the start of heating to the instant when the ignition criterion is satisfied; whereas the ignition position is defined as the axial distance from the surface to the location where the criterion of ignition is satisfied. From these definitions, criterion No. 3 (Crt. 3) always specifies an ignition position on the surface irrespective of the gravity, while criterion No. 5 (Crt. 5) does not provide any local information so that the ignition position cannot be defined. Therefore these proposed criteria are not acceptable because they contradict requirement (6) above: gravity-dependent ignition position, and requirement ( 1 ) above: local phenomenon, respectively. Although Crt. 3 and Crt. 5 are inadequate in the present study, for the purpose of comparison, the ignition position is assumed as the location of maximum gas temperature (for Crt. 3) and maximum reaction rate (for Crt. 5), respectively in the following discussions.

\section{Numerical Results}

Figures 3( a ) and (b) show that the response of ignition delay time and ignition position, respectively, to the varied gravity levels predicted by the seven criteria under the fixed ambient oxygen concentration of 0.4 . These figures clearly reveal the abrupt transition of ignition delay time at $g=0.2 \sim 0.3 \mathrm{G}$, indicating the existence of two distinct types of ignition ('tipignition' and 'inside-ignition'). In Fig. 3(a), it is observed two opposite trends in the higher gravity regime for the ignition delay time; one is the trend in terms of Crt. 2 and Crts. $4 \sim 7$ (see Fig. 3 (a-2) and Fig. $3(\mathrm{a}-4) \sim$ Fig. $3(\mathrm{a}-7))$ which show the ignition delay time increases with the gravity, and the other is in (a)
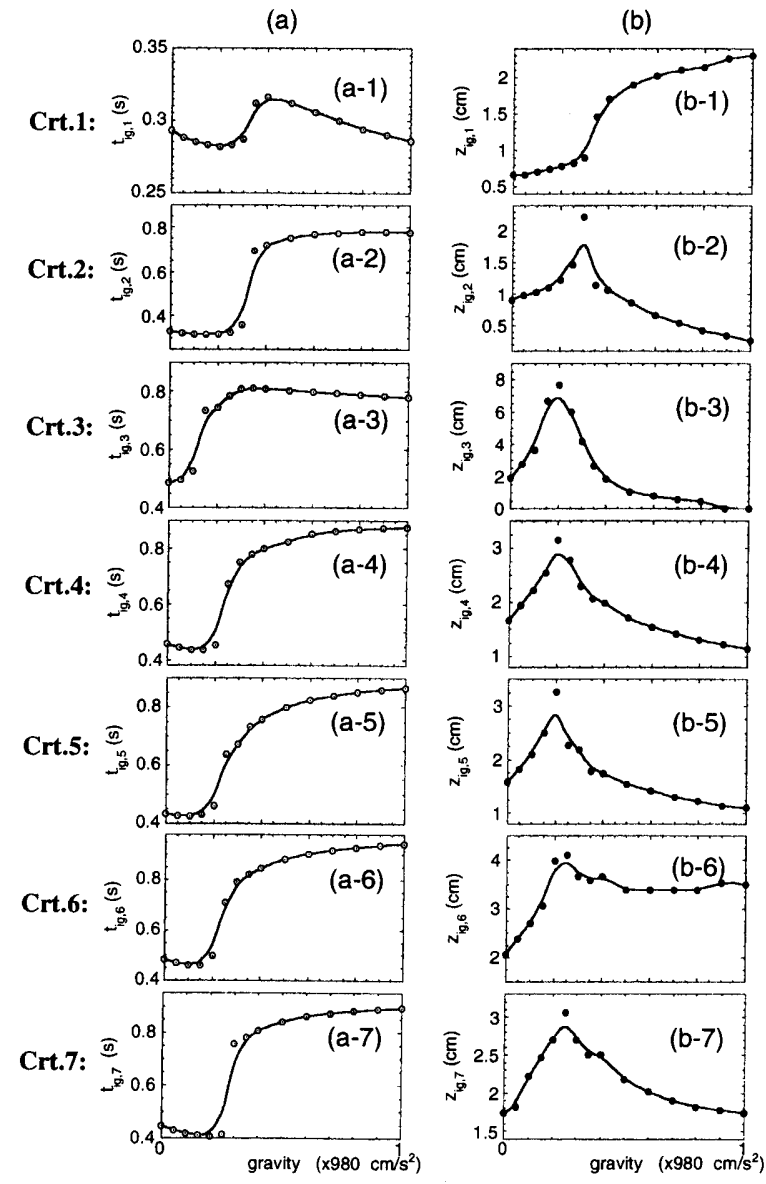

Fig. 3 The effects of gravity on (a) ignition delay and (b) ignition position for each ignition criterion (Crts. 1 7)

terms of Crt. 1 and Crt. 3 (see Fig. $3\left(\mathrm{a}^{-1}\right)$ and Fig. 3 $(\mathrm{a}-3))$ which show the ignition delay time decreases as the gravity increases. Figure $3(\mathrm{~b})$ shows a similar divergence in trends in the higher gravity regime for the ignition position. The trend in terms of Crt. 1 (Fig. 3(b-1)) shows the ignition position increases monotonically with the gravity, while Crts. $2 \sim 7$ (Fig. $3(b-2) \sim$ Fig. $3(b-7)$ ) show that the ignition position decreases as the gravity increases. It is also notified that the critical gravity which gives the abrupt transition in $t_{i g}$ and $z_{i g}$ is almost the same (nearly $g=0.25$ G) except for Crt. 1 and Crt. 2 ; these are found to be slightly larger than the others.

With the observations made so far, the requirement that there exists two types of ignition (see requirement (4) above) is satisfied in every criterion, whereas requirement (6) above: response of the ignition delay time to the gravity, is not satisfied for the proposed Crt. 1 and Crt. 3. Thus, we may safely say that Crt. 1 and Crt. 3 are inadequate criteria. We next discuss the reason why these criteria provide incorrect ignition guidelines in the light of further observation of the numerical results. 


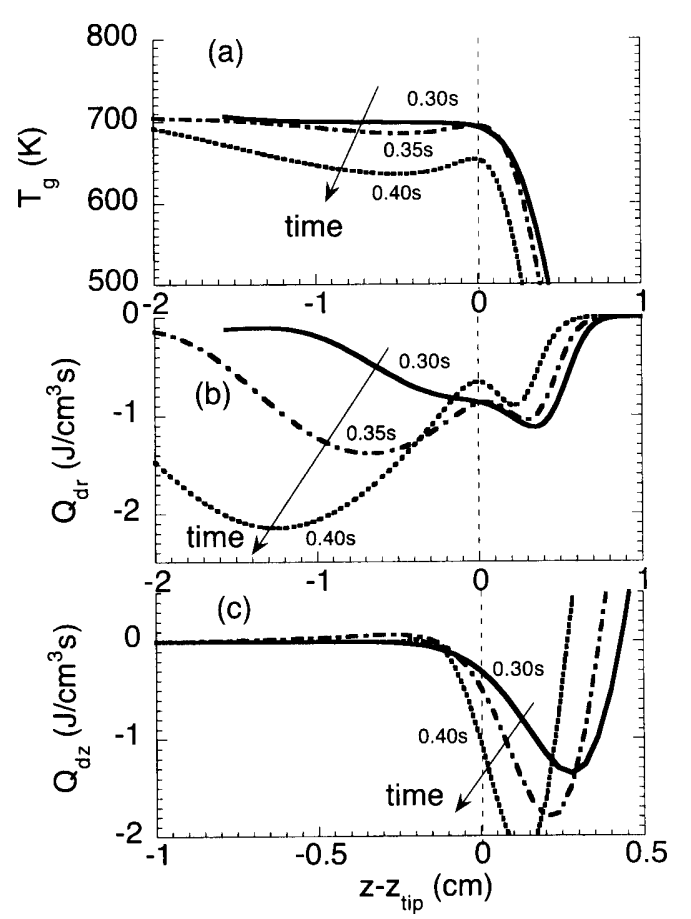

Fig. 4 Time-sequences of distributions of ( a ) temperature, (b) rate of change of the conductive heat flux in radial direction, ( $c$ ) rate of change of the conductive heat flux in axial direction from the tip of plume

\section{Discussion}

\section{1 Inadequate criteria}

6.1.1 Instant of ignition in terms of Crt. 1 As mentioned in Section 4, Crt. 1 specifies an instant when a local gas element on the axis starts to supply the heat to the surrounding ambient. In an one-dimensional model, this instantaneous local thermal peak appears only as a result of the local heat release in the gas-phase. In a multi-dimensional model, however, this is not always the case.

As seen in Figs. 2-1(b) and (c), in the presence of the gravity, the hot-gas plume is squeezed in the radial direction due to the buoyancy-induced flow. This enhances the heat loss by conduction towards the radial direction, and consequently, modifies the temperature distribution inside the plume $e^{(2),(10)}$. Figures 4 ( a ) $\sim$ ( c ) show the typical time- ${ }^{-}$hange during $t=$ $0.3 \mathrm{~s} \sim 0.4 \mathrm{~s}$ of $(\mathrm{a})$ an axial temperature distribution and the rate of change of the conductive heat flux in (b) radial/( c ) axial direction $\left(Q_{d r} / Q_{d z}: r / z\right.$ component of $\left.D \cdot\left(k D T_{g}\right)\right)$ near the plume tip on the axis at a relatively high gravity case $(g=0.4 \mathrm{G})$. The abscissa, $z^{-} z_{t i p}$, represents the relative distance from the plume tip $\left(=z_{t i p}\right.$ : defined as the location where the local reaction rate takes a peak). A negative value of $Q_{d r}$ and $Q_{d z}$ in Figs. 4 (b) and (c) indicates the heat is

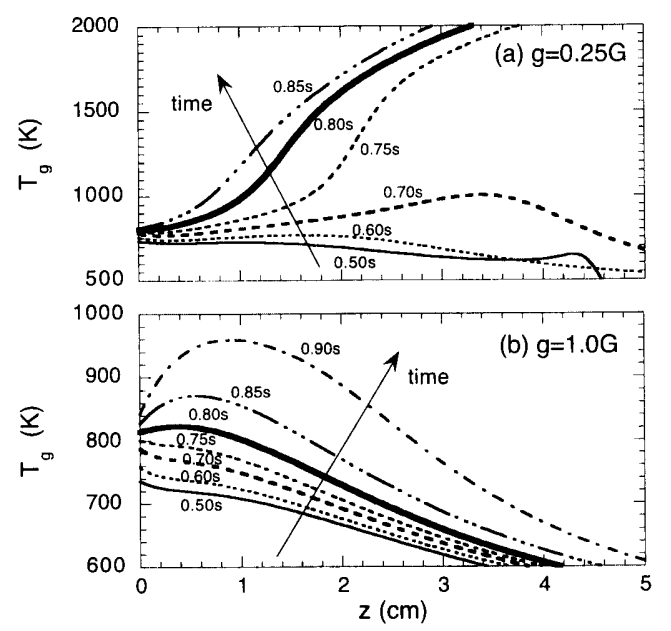

Fig. 5 The comparisons of time-sequences of distributions of temperature at ( a ) $g=0.25 \mathrm{G}$ and $(\mathrm{b})$ $g=1.0 \mathrm{G}$

removed to the ambient by the conduction (i.e. conductive heat loss). In Fig. 4 ( b ), as time goes on, it can be seen that the radial conductive heat loss is enhanced in the portion below the tip. This is due to the presence of gravity, leading to the temperature drop there to form the concave temperature distribution near the tip (see Fig. $4(\mathrm{a})$ at $t=0.35 \mathrm{~s})^{(2)}$. At this moment the zero-gradient in the axial temperature distribution is produced, i.e. the ignition in terms of Crt. 1 occurs. Once this concave temperature distribution is formed, however, the axial conductive heat loss at the top of the concave position is enhanced (see Fig. 4(c)), and consequently the temperature at the point is going to decrease (see Fig. 4 ( a ), $t=0.35 \mathrm{~s} \sim 0$. $40 \mathrm{~s}$ ) due to the excess heat loss. This behavior contradicts requirement (2) above: appearance of thermal wave after the ignition, thus criterion Crt. 1 may provide a 'false' ignition. The instantaneous concave temperature distribution at the plume tip is always observed irrespective of the level of the gravity, and the tip moves away from the surface quickly as the gravity increases (due to the buoyancy-induced flow). Therefore the ignition position in terms of Crt. 1 increases monotonically with the gravity as shown in Fig. 3(b-1).

6.1.2 Instant of ignition in terms of Crt. 3 Figures 5(a) and (b) show that the typical timechange of axial temperature distributions in two different gravity conditions ( $(\mathrm{a}) g=0.25 \mathrm{G}$ and ( $\mathrm{b}$ ) $g=1.0 \mathrm{G})$. Note that, the ignition delay times in terms of Crt. 3 for these two gravity conditions remain almost the same, $t=0.78 \mathrm{~s}$, as is seen in Fig. $3(\mathrm{a}-3)$. In Fig. 5, however, the maximum temperatures at the instant of the ignition are found to be very much different for these cases; over $2000 \mathrm{~K}$ for $g=0.25 \mathrm{G}$ and around $800 \mathrm{~K}$ for $g=1.0 \mathrm{G}$. Especially for the 
former case, the temperature is too high to characterize the ignition; it is almost the fully developed flame temperature. They apparently do not describe the same instantaneous phenomenon. The reason why Crt. 3 tends to produce the unrealistic ignition instant may be related to the difference of the location where the incipient flame appears in various gravity conditions. Here, the incipient flame means the temperature peak position which finally develops into the propagating thermal wave (i.e. ideal ignition position') ; it is located away from the surface for $g=0.25$ $\mathrm{G}$ at $t=0.7 \mathrm{~s}$, whereas it is located near the surface for $g=1.0 \mathrm{G}$ at $t=0.8 \mathrm{~s}$ as seen in Figs. 2 and 5.

Once the incipient flame appears away from the surface (in Fig. 5( a ), $g=0.25 \mathrm{G}, t=0.7 \mathrm{~s}$ at $z=3.5 \mathrm{~cm}$ ), the heat conduction from there toward the surface (i.e. downward conductive heat transport) occurs. On the other hand, the incipient flame induces upward buoyancy-induced flow so that the upward convective heat transport from there is enhanced. These two heat transports compete with each other, and consequently the appearance of the incipient flame does not affect immediately the thermal status of the surface. In another word, the surface thermal status may not respond quickly to the change of the thermal status in gas-phase when the incipient flame appears away from the surface. On the contrary, when the incipient flame appears near the surface (in Fig. 5( b ), $g=1.0 \mathrm{G}$, $t=0.8 \mathrm{~s}$ at $z=0.5 \mathrm{~cm}$ ), the buoyancy-induced flow will not play a significant role at that location so that the thermal status of the gas-phase immediately affects the surface thermal status by conduction. Therefore it is concluded that the ignition in terms of Crt. 3 provides inadequate instant of the ignition because of its strong dependency on the location of the incipient flame, i.e. the ideal ignition position. It is interesting to note that the strong dependency of the ignition delay time in terms of Crt. 3 on the ideal ignition position can be found in the comparison of Fig. $3(\mathrm{a}-3)$ and Figs. $3(b-2) \sim(b-7)$, indicating the same dependence on gravity; they increase with gravity in the lower gravity regime and decrease at higher gravity levels.

In view of the above observations, it is understood that criterion Crt. 1 and Crt. 3 provide inadequate instant of the ignition due to the larger heat loss to the radial direction induced by the gravity and the enhanced upward convective heat transport by the buoyancy-induced flow, respectively. It is noted that these reasons are related with the buoyancy-induced flow, i.e. the multi-dimensional transport process. Therefore these criteria cannot be applied to the ignition problems where the multi-dimensional transport processes come to play, even though they can reasonably describe the instant of the ignition in one-dimensional case.

6.1.3 Difference of critical gravity in terms of Crt. 2 The ignition proposed in terms of Crt. 2 can be correct when the surface temperature strongly controls the gas-phase ignition and that there is no chance for the transport events to be involved. Once the transport events in gas-phase come to play a role in the ignition process, however, the criterion of Crt. 2 would be no longer valid. As example, consider the non-monotonic behavior of the temperature at the plume tip in $g=0.25 \mathrm{G}$ as described in Section 3.2 (see Fig. 2-3); the temperature at the plume tip (located away from the surface) monotonically increases with time at first, but then decreases. During this event, on the other hand, the surface temperature increases monotonically ${ }^{(2)}$. This indicates that the thermal status of the surface cannot control every phenomenon in the gas-phase, especially one occurring far from the surface. Therefore it is not surprising that the local gas temperature can exceed the surface temperature instantaneously (i.e. the ignition in terms of Crt. 2 occurs), but then declines when the critical gravity is reached. Finally it is concluded that Crt. 2 may provide an inadequate instant of ignition near the critical gravity (showing the abrupt transition from 'tip-ignition' to 'inside-ignition') where the thermal status of the plume tip plays a key role on whether the ignition occurs or not. This may be consistent with some findings in the earlier experimental and analytical studies; it has been reported that the surface temperature does not always control the gas-phase ignition ${ }^{(18)}$ and suggested that the thermal status in the gas-phase, rather than that in the solid-phase, plays a significant role in the process leading to the gas-phase ignition $^{(19)}$. Therefore it can be concluded that criterion Crt. 2 is not appropriate in general.

Thus, criteria based on temperature alone (i.e. the local gas-temperature or the surface temperature) cannot provide the correct qualitative ignition behavior; they will predict a 'false' ignition occasionally. Some quantities other than temperature have to be chosen to describe an appropriate criterion of the ignition.

\subsection{Determination of appropriate criterion of ignition}

In view of the discussion to this point, it is found that Crt. $1 \sim$ Crt. 3 are inadequate for a general prediction of ignition, and Crt. 5 is also not useful because the local information is not included. In this section we discuss the remaining proposed criteria; Crt. 4 , Crt. 6 and Crt. 7.

Note that these criteria all have a common form ; ignition is defined as the instance when "(physical 


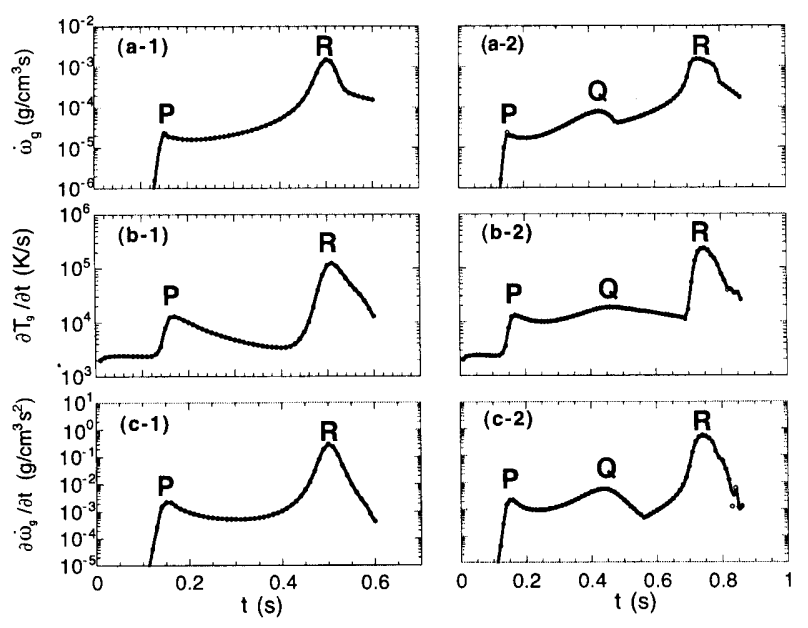

Fig. 6 The comparisons of time-history of (a) maximum reaction rate, $(b)$ maximum time-change of temperature and $(\mathrm{c})$ maximum time-change of reaction rate for each distinct type of ignition (tip ignition : left, inside ignition : right)

variables) exceeds (a certain constant: $C_{i g}$ )". The previous discussions show that all of the three criteria with arbitrary-selected $C_{i g}$ satisfy requirements ( 1$) \sim(6)$ above in various gravity levels under the fixed ambient oxygen concentration of 0.4 . In the light of the last requirement (7) above; independence from parameters, these criteria are examined in various gravity levels and various ambient oxygen concentrations. In the following, we focus on how to determine $C_{i g}$ which can be valid in the wide range of gravity levels and ambient oxygen concentrations.

Figure 6 shows the typical time-change of the maximum of axial distributions on the axis of (a) $\dot{\omega}_{g}$, (b) $\partial T_{g} / \partial t$, (c) $\partial \dot{\omega}_{g} / \partial t$ during the process of ignition for distinct two types of ignition. As is seen in the figure, there are two peaks in the tip ignition case whereas three peaks in the inside ignition case. Each peak corresponds to the following event (named $\mathrm{P}, \mathrm{Q}, \mathrm{R}$ respectively) ;

* The instant when the decomposed reaction of solid-phase starts to evolve $(\sim 0.15 \mathrm{~s}): \mathrm{P}$

* The instant when the non-monotonic event (i.e. false ignition) occurs at the plume tip: $Q$

* The instant when the thermal wave following the true ignition appears: $\mathrm{R}$.

Now, the numerical values, $C_{i g}$, for each criterion must satisfy the following inequalities ;

$$
\max (\mathrm{P}, \mathrm{Q})<C_{i g}<\min (\mathrm{R})
$$

where $\max (\mathrm{P}, \mathrm{Q})$ and $\min (\mathrm{R})$ represent, respectively, the larger one of the maximum values of $\mathrm{P}$ or $\mathrm{Q}$ and the minimum value of $\mathrm{R}$ in the varied range of the parameters; gravity level (zero- $g$ to normal- $g$ ) and ambient oxygen concentration (zero to one hundred percent). The first inequality is necessary to avoid the
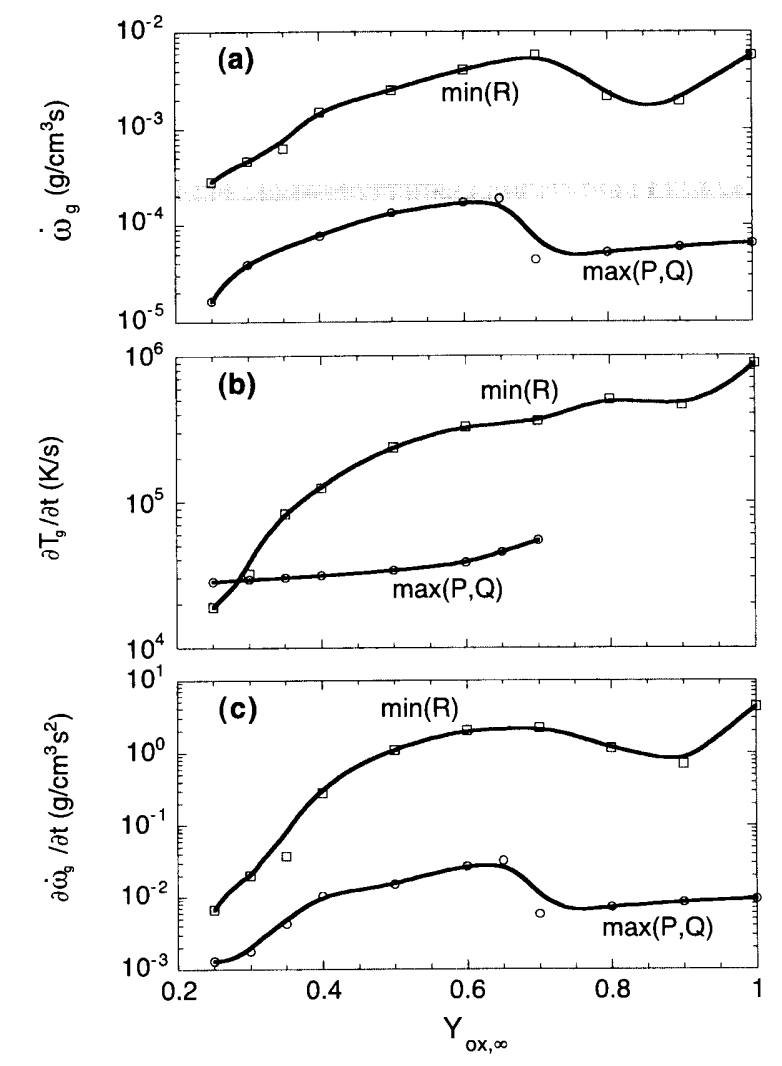

Fig. 7 The comparisons of available range of each ignition criterion in various ambient oxygen fields; (a) maximum reaction rate, (b) maximum timechange of temperature, (c) maximum timechange of reaction rate

'false' ignition, while the second inequality is required to recognize the 'true' ignition.

Figure 7 shows that how the $\max (\mathrm{P}, \mathrm{Q})$ and the $\min (\mathrm{R})$ change with the ambient oxygen concentrations for each case of (a) $\dot{\omega}_{g}$, (b) $\partial T_{g} / \partial t$, ( c) $\partial \dot{\omega}_{g} / \partial t$. Note that the plotted $\max (\mathrm{P}, \mathrm{Q})$ value in each specified ambient oxygen concentration is evaluated in various gravity levels and selected as its maximum value. Therefore there exist no other higher values than the plotted $\max (\mathrm{P}, \mathrm{Q})$ in the specified ambient oxygen concentration. In the same manner, the plotted $\min (R)$ value represents the minimum value in the varied gravity levels under the specified ambient oxygen concentration.

In Fig. 7(b): $\partial T_{g} / \partial t$ shows that the inequality $\max (\mathrm{P}, \mathrm{Q})<\min (\mathrm{R})$ is not satisfied in the low ambient oxygen concentration regime. This is because the ignition event in this regime is relatively slow (i.e. $\min (R)$ is small), and consequently, the increasing rate of the gas-phase temperature at the 'true' ignition becomes occasionally smaller than that of the decomposition stage of solid fuel in vicinity of the surface (i.e. $\max (\mathrm{P})$ ). Therefore this criterion of ignition (Crt.6) is valid only in the higher ambient 
oxygen concentration regime leading to the abrupt thermal explosion; this is inadequate as a general criterion of the ignition.

In Fig. $7(\mathrm{c}): \partial \dot{\omega}_{g} / \partial t$, it shows that the inequality of $\max (P, Q)<\min (R)$ is satisfied in all conditions. Note that, however, there is no universal $C_{i g}$ value in the whole range of ambient oxygen concentration. Therefore it can be concluded that this criterion (Crt. 7) is also inadequate for general use.

Finally it can be seen that, for criterion of Crt. 4 : $\left(\dot{\omega}_{g}\right)$ only, there exists a universal $C_{i g}$ value in the whole range of ambient oxygen concentration, that satisfies requirement ( 7 ) above. Appropriate values for $C_{i g}$ can be found in the shaded region in Fig. 7 ( a ) at around $2.0 \times 10^{-4} \mathrm{~g} /\left(\mathrm{cm}^{3} \mathrm{~s}\right)$. This finding implies the following important conclusion; Since the heat release rate is directly related to the reaction rate, if the value for $C_{i g}$ represents the magnitude of local heat loss rate, Crt. 4 is basically the same as the thermal explosion theory; ignition is determined as the instant when the local heat release rate (equivalent to local reaction rate) exceeds the local heat loss rate. It should be emphasize that the concept of the thermal explosion is still valid even under such a complex ignition process with multi-dimensional gravity effects. Although the numerical value of $C_{i g}$ may depend on the specific problem under consideration, as long as an appropriate $C_{i g}$ is chosen, Crt. 4 is capable to be the most appropriate criterion for general ignition problems.

\section{Concluding Remarks}

We have studied numerically the appropriate criterion for spontaneous ignition that can be applied to the process in multi-dimensional configuration with various gravity levels and ambient oxygen concentrations. Seven representative ignition criteria, often used in the vast of ignition studies, were tested to determine if they can predict correctly the ignition behavior in a widely varied range of physical parameters. Detailed discussions of the predicted dependence of the ignition characteristics (i.e. ignition delay time and ignition position) on the gravity level and the ambient oxygen concentration have led to the following conclusions ;

1. Some criteria cannot predict qualitatively valid ignition characteristics in multi-dimensional ignition processes, although they do in one-dimensional ignition processes.

2. The criteria that are occasionally satisfied in the course of multi-dimensional mass, heat and momentum transport processes should not be taken as the general criterion.

3. The criterion based on the condition that the local heat release rate exceeds a certain critical value is found to be the most appropriate one if we can select an adequate value for that constant. This implies that the concept of thermal explosion theory can still be applied locally to express an ignition even if it occurs in complex, multi-dimensional heat and mass transport processes.

\section{Acknowledgments}

Authors would like to express our sincere thanks to Dr. Howard Baum for his valuable suggestions and help in polishing the English.

\section{References}

(1) Nakamura, Y., Kushida, G., Yamashita, H. and Takeno, T., A Numerical Study of Gravity Effects on Spontaneous Ignition, Proc. of the 1998 Technical Meeting of the Central States Section of The Combustion Institute, (1998), pp. 128-133.

(2) Nakamura, Y., Yamashita, H., Takeno, T. and Kushida, G., Effects of Gravity and Ambient Oxygen on a Gas-Phase Ignition over a Heated Solid Fuel, Combust. Flame, Vol.120, No. 1 (2000), pp. 34-48.

(3) Nakamura, Y., Kushida, G., Yamashita, H. and Takeno, T., A Behavior of Initial Flame Kernel in Process of Irradiated Ignition of Solid Fuel (Effects of Natural Convection on Gas-Phase Ignition Processes), Proc. of Thirty-Third Japanese Symposium on Combustion, (in Japanese), (1995), pp. 608-610.

(4) Adomeit, G., Ignition of Gases at Hot Surfaces under Non Steady-State Conditions, Tenth Sympo. (Int'1) on Combust., The Combustion Institute, Pittsburgh, pp. 237-243 (1965).

(5) Semenov, N.N., Some Problems in Chemical Kinetics and Reactivity, Vol. II (1959), Chapt. VIII, Princeton University Press, Princeton.

(6) Frank-Kamenetskii, D.A., Diffusion and Heat Transfer in Chemical Kinetics, 2nd. Ed., (1969), Prenum Press.

( 7 ) Kashiwagi, T., A Radiative Ignition Model of a Solid Fuel, Combust. Sci. Tech., Vol. 8 (1974), pp. 225-236.

(8) Kumar, R.K., Gas Phase Ignition of Composite Solid Propellant Subjected to Radiant Heating, Combust. Sci. Tech., Vol. 30 (1983), pp. 273-288.

(9) Gandhi, P.D. and Kanury, A.M., Criterion for Spontaneous Ignition of Radiantly Heated Organic Solid, Combust. Sci. Tech., Vol. 50 (1986), pp. 233-254.

(10) Nakamura, Y., Kushida, G., Yamashita, H. and Takeno, T., Effects of Natural Convection on Self-Ignition of Solid Fuel Heated by External Radiation, Trans. Jpn. Soc. Mech. Eng., ( in Japanese), Vol. 62, No. 5, B (1996), pp. 2758-2766.

(11) Amos, B. and Fernandez-Pello, A.C., Model of the Ignition and Flame Development on a Vaporizing 
Combustible Surface in a Stagnation Point Flow, Combust. Sci. Tech., Vol. 62 (1988), pp. 331-343.

(12) Zhang, D.K., Hills, P.C., Zheng, C., Wall, T.F. and Samson, P., Fibre Optic Ignition of Combustible Gas Mixtures by the Radiative Heating of Small Particle, Twenty-Fourth Sympo. (Int'l) on Combust., (1992), pp. 1761-1767, The Combustion Institute, Pittsburgh.

(13) Kashiwagi, T., MacDonald, B.W., Isoda, H. and Summerfield, M., Ignition of a Solid Polymeric Fuel in a Hot Oxidizing Gas Stream, Thirteenth Sympo. (Int'l) on Combust., (1971), pp. 1073-1086, The Combustion Institute, Pittsburgh.

(14) Park, S.H. and Tien, C.L., Radiation Induced Ignition of Porous Solid Fuels, Combust. Sci. Tech., Vol. 95 (1994), pp. 173-192.

(15) Beckel, S.A. and Matthews, R.D., Ignition of Polyoxymethylene, Combust. Flame, Vol. 57 (1984), pp. 71-86.
(16) Di Blasi, C., Crescitelli, S., Russo, G. and Cinque, G., Numerical Model of Ignition Processes of Polymeric Materials including Gas-Phase Absorption of Radiation, Combust. Flame, Vol. 83 (1991), pp. 333-344.

(17) Kashiwagi, T. and Nambu, H., Global Kinetics Constants for Thermal Oxidative Degradation of a Cellulosic Paper, Combust. Flame, Vol. 88 (1992), pp. 345-368.

(18) Niioka, T., Takahashi, M. and Izumikawa, M., Gas-Phase Ignition of a Solid Fuel in a Hot Stagnation-Point Flow, Eighteenth Sympo. (Int'l) on Combust., (1981), pp. 741-747, The Combustion Institute, Pittsburgh.

(19) Kindelan, M. and Williams, F.A., Gas-Phase Ignition of a Solid with In-Depth Absorption of Radiation, Combust. Sci. Tech., Vol. 16 (1977), pp. 4758 . 